\title{
Influence of Medical Compression Stockings on Skin Hydration in Mainly Health Care Givers with Occupational Leg Symptoms and Edema
}

\author{
Marina Mayer-Yousifa ${ }^{a}$ Wolfgang Konschake ${ }^{a}$ Hermann Haase $^{a}$ \\ Michael Jünger $^{a}$ Helene Riebe $^{a}$ \\ ${ }^{a}$ Department of Dermatology, Universitätsmedizin Greifswald, Greifswald, Germany
}

\section{Keywords}

Chronic venous insufficiency · Wear comfort - Quality of life $\cdot$ Compression therapy $\cdot$ Integrated skin care

\begin{abstract}
Background and Objective: Although compression therapy is a very effective therapy in reducing stasis-induced complaints, the wearing comfort is not always as requested. Most frequent problems are dryness of the skin and itching. This randomized, cross-over trial investigated 33 healthy participants and compared 2 different medical compression stockings: conventional stockings (type $A=M C S$ ) versus compression stockings with integrated care emulsion (type $B=$ MCSSkC). Methods: Participants were divided into 2 cohorts. Both compression types were worn one after the other, 1 week each. The cohorts were named according to the sequence of the wearing periods (cohort AB/BA). Primary outcome: skin hydration. Secondary outcomes: transepidermal water loss (TEWL), skin roughness, leg volume, interface pressure, and questionnaires about stasis-induced complaints and wearing comfort. Results: Skin hydration: significant reduction after wearing MCS in both cohorts $(p<0.001)$; preservation of skin moisture after wearing MCS-SkC $(p=$
\end{abstract}

karger@karger.com www.karger.com/spp

Karger $\stackrel{\text { ' }}{5}$

GOPEN ACCESS
(C) 2021 The Author(s)

Published by S. Karger AG, Basel

This is an Open Access article licensed under the Creative Commons Attribution-NonCommercial-4.0 International License (CC BY-NC) (http://www.karger.com/Services/OpenAccessLicense), applicable to the online version of the article only. Usage and distribution for commercial purposes requires written permission.
0.546 and $p=0.1631)$. TEWL: significant increase after wearing MCS ( $p=0.007$ and $p=0.0031)$; significant reduction by wearing MCS-SkC ( $p=0.006$ and $p=0.0005)$. Skin roughness: significant increase after wearing MCS $(p=0.0015$ and $p=$ $0.010)$, and nonsignificant decrease of skin roughness after wearing MCS-SkC ( $p=0.933$ and $p=0.4570)$. Leg volume: significantly reduced with both stockings $(p=0.004$ and $p=$ 0.0047). Regarding stasis-induced complaints, both stockings achieved good results. Conclusions: Both compression stockings are appropriate to reduce leg edema and minimize leg symptoms. MCS-SkC helps to obtain the natural skin barrier function in preserving the epidermal water content and reducing the TEWL.

(C) 2021 The Author(s)

Published by S. Karger AG, Basel

\section{Introduction}

Leg pain and its increased prevalence in the general population is a major issue of current studies. The crosssectional Bonn Vein Study revealed that of 3,072 tested persons, every sixth male and almost every second female are suffering from leg swelling and other symptoms such as feeling of tension and heaviness [1]. In a cross-over 
trial conducted in 2009/2010, Blazek et al. [2] have shown that individuals who work in a profession requiring prolonged standing habitually experience leg symptoms. Even if such symptoms are mostly connected to venous diseases, the study confirmed that there was a considerable ratio of individuals whose veins did not show any pathological changes despite leg swelling and feeling of heaviness. Thus, the occurrence of symptoms did not imply the presence of a manifest venous disorder [2]. Regarding ICD-10 criteria, occupational leg edema is not a defined disease although it causes different symptoms like leg swelling, feeling of heaviness, and tension as well as mental problems, which justify a basic therapy with compression stockings. The above-citied study by Blazek et al. [2] demonstrated that a treatment with compression stockings Ccl. 1 had a significant influence on occupational leg symptoms. Including a cohort of 108 persons, the trial showed that not only the feeling of tension and heaviness could be reduced by $12 \%$, but also there was a decrease of leg volume by an average of $19 \mathrm{~mL}$ [2]. However, the effectiveness of compression therapy is mainly determined by the individual's compliance which varies with the manifestation of side effects like dryness of the skin, itching, and constriction. In 2009, Reich-Schupke et al. [3] questioned 110 participants about their quality of life under compression therapy. Merely $29.1 \%$ assessed the therapy as comfortable, whereas dryness of the skin (58.8\%) and itching (32.7\%) were the main adverse events [3]. These items are clinical signs of textile-associated dehydration of the skin and indicate an impaired skin barrier function. An essential factor in maintaining a normal skin barrier is the ability to retain water in the stratum corneum [4]. Particularly, there are 2 factors which are important in maintaining an optimal level of hydration within the stratum corneum [5]. First of all, polar lipids (cholesterol, fatty acids, and ceramides) which fill in the intercellular spaces between the corneocytes and thus prevent transepidermal water loss (TEWL) $[5,6]$. Secondly, and equally important, is the so-called natural moisturizing factor (NMF) which is a mixture of hydrophilic compounds (amino acids, lactic acid, and urea) and therefore able to bind water [5].

The aim of this randomized trial was to examine the influence of 2 different types of medical compression hosiery on skin hydration, TEWL, and skin roughness being parameters of the skin barrier function. In this cross-over design, all participants, mainly health care givers, had to wear a conventional compression hosiery (MCS = stocking type A) and a medical compression system with an integrated skin care emulsion (MCS-SkC = stocking type B).

\section{Materials and Methods}

\section{Study Design}

In this randomized, double-blind, prospective cross-over trial, exclusively healthy people have been included. Therefore, the volunteers, mainly medical health care givers, were screened regarding their suitability before starting this trial. Qualified participants were divided into 2 randomized cohorts. Both groups had to wear 2 types of compression stockings in reverse order for 1 week each. Cohort $\mathrm{AB}$ started wearing standard medical compression stockings (A) and switched to the medical compression stockings with integrated skin care emulsion (B). Cohort BA started with medical compression stockings with integrated skin care emulsion (B) and switched to the conventional compression stockings (A). The primary outcome was the skin hydration influenced by the different types of compression stockings. Secondary outcomes were the assessment of leg volume, TEWL, skin roughness, interface pressure, and questionnaires interrogating stasis-induced complaints and wearing comfort. The study was registered in the German clinical trials register (DRKS-ID: DRKS00010661).

\section{Participants}

In this prospective cross-over trial, a cohort of 33 participants (25 females and 8 males) with an average age of 33.97 years were included. Inclusion criteria comprised healthy female and male volunteers (aged between 18 and 80 years), mainly health care givers, without any objectified venous disease, who spend long periods standing in their daily life ( $>8 \mathrm{~h}$ ) and develop occasional leg symptoms like feeling of heaviness, tension, and evening edema. Exclusion criteria were characterized as follows: CVI-related leg edema, deep vein thrombosis, arterial occlusive disease, diabetes mellitus with neuropathy and/or peripheral circulatory disorder, simultaneous participation in another trial, or known pregnancy. All participants agreed to wear the 2 different types of compression hosiery (medical compression stockings, Ccl. 1, AD or AG, with and without care emulsion) for at least $8 \mathrm{~h} /$ day. Noncompliance resulted in an immediate disqualification.

\section{Compression Materials}

In a randomized order, 2 different types of elastic compression stockings available in lengths $\mathrm{AD}$ or AG were tested (Bauerfeind AG, Zeulenroda, Germany). Both stocking types had a maximal interface pressure exerted at the area of the ankle (defined as B point) with a declining pressure from distal to proximal. Compression stockings MCS and MCS-SkC were RAL tested (Reichs-Ausschuss für Lieferbedingungen, Sankt Augustin, Germany) and approved medical devices (CE) (Table 1).

\section{Protocol and Procedure}

The participants were divided into 2 cohorts. One group started wearing MCS, while the other group started with MCS-SkC. The allocation to one or another cohort was randomized. After a wear period of 1 week, the participants switched to the second type of hosiery which had to be worn another week. Taken as a whole, this implied a 2 -week test period. Both wearing periods were accomplished consecutively. The final investigation of wearing period 1 was the initial investigation for wearing period 2 simultaneously. Each type of compression stocking was worn at least $8 \mathrm{~h}$ per day. For clarity of the cross-over design, the 2 cohorts were named after the sequence of wearing periods A and B (Fig. 1).
Mayer-Yousif/Konschake/Haase/Jünger/ Riebe 


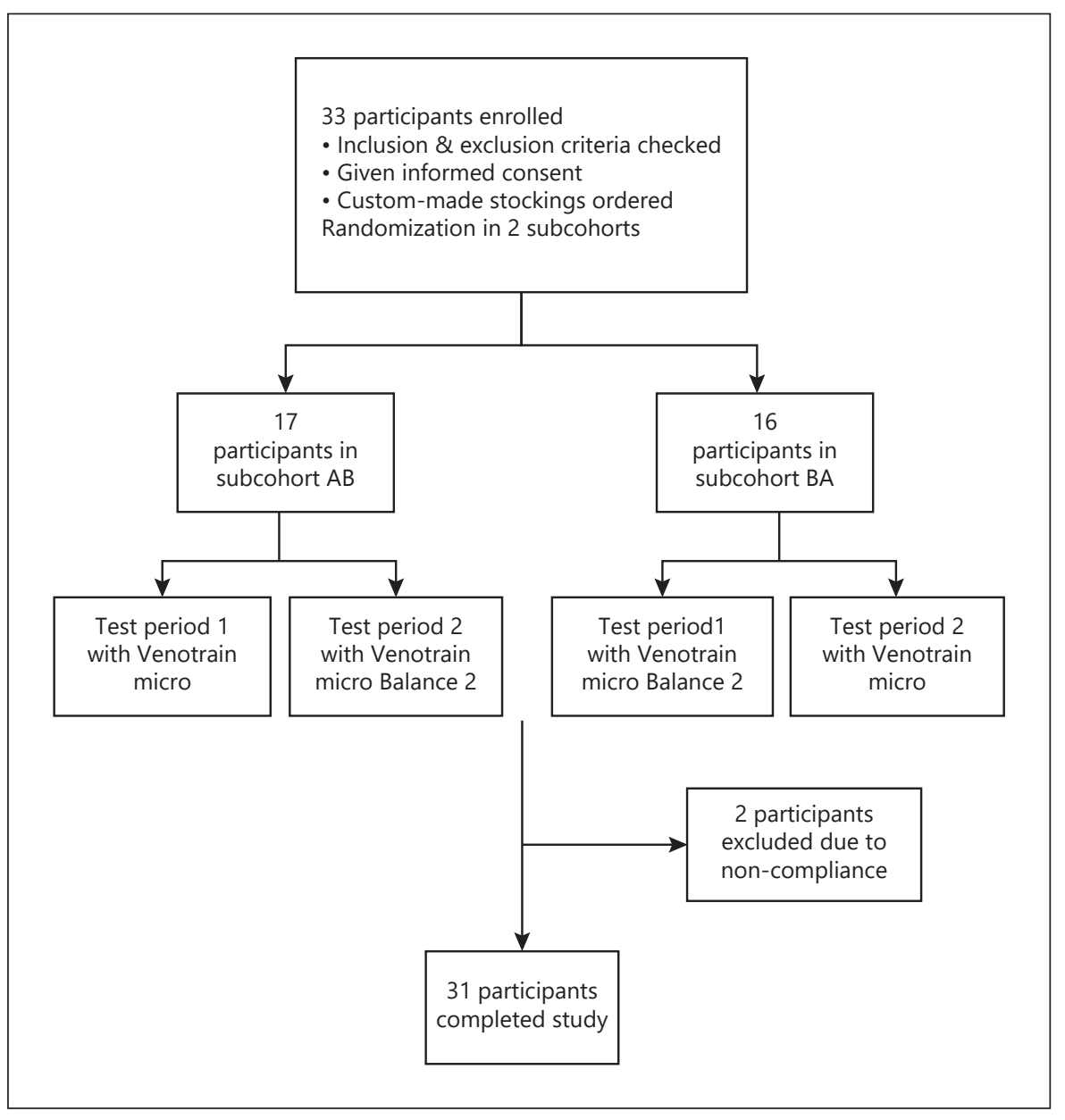

Fig. 1. Consort diagram.

All visits and measurements were performed by the same investigator and took place in temperature-controlled locations. In order to receive comparable data, all investigations for each volunteer were scheduled at the same time of a day.

\section{Screening Visit}

Before starting this trial, the volunteers had to be screened regarding their suitability. Therefore, all inclusion and exclusion criteria have been reviewed. Besides anamnesis and general physical examination, the current condition of the participants' legs regarding the presence of venous diseases (e.g., CVI) has been assessed not only clinically but also by objectifying the findings by testing the venous function through digital photoplethysmography using DPPG Vasoquant $1000^{\circledR}$ (Elcat $\left.\mathrm{GmbH}\right)$. Once a volunteer was qualified, an informed consent had to be signed in order to take part in this prospective trial. Following this, the leg volume of each participant was defined by using Bodytronic ${ }^{\circledR} 600$. On the basis of the measured data, the compression stockings were ordered using an online ordering system.

Visit (Day) 1

At a maximum of 7 days ahead of the first wearing period, the basic status of each participant had to be determined. This comprised anamnesis, physical examination, and a photodocumenta-
Table 1. Both types of used medical compression stockings

\begin{tabular}{|c|c|}
\hline Venotrain ${ }^{\circledR}$ micro & Venotrain ${ }^{\circledR}$ micro balance 2 \\
\hline Elastic compression stockings, & Elastic compression stockings \\
\hline Ccl. 1 with interface pressure & Ccl. 1 with interface pressure of \\
\hline of $18-21 \mathrm{~mm} \mathrm{Hg}$ & $18-21 \mathrm{~mm} \mathrm{Hg}$ and integrated \\
\hline & care emulsion produced by \\
\hline & Sebapharma GmbH \& Co. \\
\hline & $\begin{array}{l}\text { Main ingredients: natural oils, } \\
\text { vitamin E derivate, and urea }\end{array}$ \\
\hline
\end{tabular}

Ccl. 1, compression class 1 .

tion displaying the current condition of the studied leg (left/right). Additionally, the following measurements were carried out: leg volume by use of Bodytronic ${ }^{\circledR} 600$, skin hydration by use of Corneometer ${ }^{\circledR}$ CM 825, TEWL by use of Tewameter ${ }^{\circledR}$ TM 300, skin roughness by use of FOITS, and compression pressure by use of PicoPress ${ }^{\circledR}$. The measurement of skin hydration was performed 4 times and the measurement of the TEWL 2 times. The results have been averaged afterward. 
Table 2. Standard values of skin moisture as characterized by Courage \& Khazaka Electronic GmbH

\begin{tabular}{lc}
\hline Skin moisture & AU \\
\hline Very dry skin & $<30$ \\
Dry skin & $30-40$ \\
Sufficiently moisturized & $>40$ \\
\hline
\end{tabular}

Table 3. Standard values of TEWL as characterized by Courage \& Khazaka Electronic GmbH

\begin{tabular}{lr}
\hline TEWL & $\mathrm{g} / \mathrm{m}^{2} \mathrm{~h}$ \\
\hline Very healthy skin & $0-10$ \\
Healthy skin & $10-15$ \\
Normal skin & $15-25$ \\
Strained skin & $25-30$ \\
Critical skin condition & $>30$ \\
\hline
\end{tabular}

TEWL, transepidermal water loss.

According to the affiliation to one of the 2 cohorts, the first pair of stockings were handed out in a randomized, double-blinded way (cohort A/B). Each participant received an initial instruction in the handling of compression hosiery. This included the guidance how to pull them on correctly as well as the adequate cleansing with a uniform detergent. Additionally, the participants were instructed to use a $\mathrm{pH}$-neutral shower gel during the wearing period which was also handed out. Questionnaires interrogating stasis-induced complaints and wearing comfort were handed out and elucidated to each participant.

\section{Visit (Day) 7}

This visit occurred at day 7 of the first wear period, and it was defined as the endpoint of wear period 1. Again, a short anamnesis, physical examination, and photodocumentation were performed in order to assess variances in the current health status and skin condition compared to day 1 . The measurements of leg volume, skin hydration, TEWL, skin roughness, and compression pressure were repeated as well. At this, the received data were set as baseline for wear period 2 which had to be started the very next day after finishing with test period 1. According to the affiliation to one of the 2 cohorts, the second pair of stockings were handed out in a randomized, double-blinded way (cohort A/B).

\section{Visit (Day) 14}

It is defined as the endpoint of the second period after 7 days of wearing. A short anamnesis, physical examination, and photodocumentation in order to assess variances in the current health status and skin condition compared to day 7 were carried out. Additionally, the following measurements were repeated: leg volume, skin hydration, TEWL, skin roughness, and compression pressure. At last, the questionnaires were collected and looked at if completed properly.

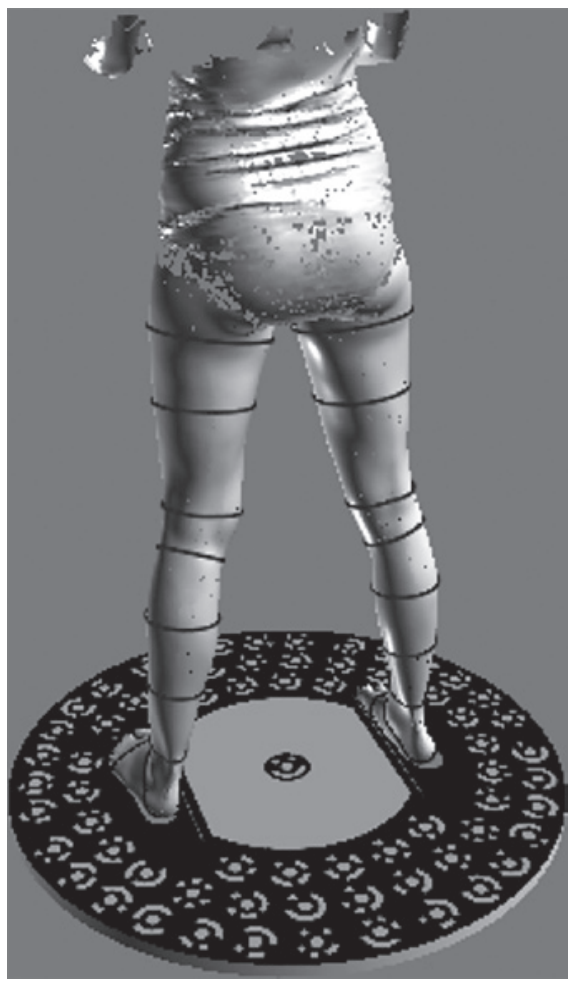

Fig. 2. Participants standing on the rotating platform of Bodytronic ${ }^{\circledR}$ while producing a 3D image.

Measurements of skin hydration were performed by using Corneometer ${ }^{\circledR}$ CM 825 (Courage \& Khazaka Electronic, Köln, Germany). The measurement is based on the potential alteration of capacity of a dielectric medium such as the stratum corneum which changes its dielectrical features depending on its moisture content. Therefore, the probe head is equipped with a metallic track which emits an electrical scatterfield and acts as a capacitor. Depending on the water content of the sample, the capacitor changes its capacitance. The measurement uses arbitrary units (AUs) between 0 and 130. The higher the quantity of AUs, the more water is retained within the measured skin area. The standard values are characterized as is shown in Table 2. These standard values are not strictly defined. All values represent approximations only. The measured values depend on different factors like temperature, humidity, geographical location, season, and gender. In order to avoid the influence of deeper skin layers, the measurement depth is very small $(10-20 \mu \mathrm{m})$ [7].

TEWL is considered to be an appropriate parameter for the barrier function of the skin and has been measured by using Tewameter $^{\circledR}$ TM 300 (Courage \& Khazaka Electronic, Köln). In order to receive data, the probe measures the epidermal water evaporation which increases with damaged skin. Therefore, 2 pairs of sensors for temperature and relative humidity are located inside a hollow cylinder which creates a homogenous diffusion zone. The physical basis for this open chamber measurement is the diffusion law described by Adolf Fick. The diffusion coefficient is defined as a mass of substance which diffuses through a unified surface in a unified time $\left(\mathrm{g} / \mathrm{m}^{2} \mathrm{~h}\right)$ [8]. Table 3 can be used as an interpretation help. 
Table 4. Stasis-induced complaints and wearing comfort during wearing periods 1 and 2

\begin{tabular}{ll}
\hline Feeling of constriction and tightness & Constriction, generally \\
& Constriction, knee area \\
& Constriction, ankle area \\
& Tightness, generally \\
& Tightness, knee area \\
& Tightness, ankle area \\
\hline Skin changes & Skin dryness \\
& Skin roughness \\
& Skin rash \\
& Skin flaking \\
\hline Aberrant sensations & Sensation of coldness \\
& Sensation of warmth \\
& Sensation of tingling \\
& Pain \\
Sensation of burning & Itch \\
\hline
\end{tabular}

Sweating beneath compression hosiery

In order to assess the skin surface (e.g., roughness), a fast optical in vivo topometry of human skin (FOITS) has been used. Dermatop $^{\circledR}$ (Breuckmann GmbH, Meersburg, Germany) analyzes the microstructure of human skin and produces a 3-dimensional image. Skin irregularities can be defined in the range of $\mathrm{mm}^{3}$ by measuring a positive and negative volume. Therefore, the system consisting of a CCD camera and projection unit uses a strip analysis, a combination of phase shifting and the gray-code method. By means of both techniques, the deviation of the investigated area as compared to a plane surface can be calculated. Based on that surface, the volume of bulges and indentations can be added and indicated as positive $\left(V_{+}\right)$and negative $\left(V_{-}\right)$volume. The measured volume $V_{+} / V_{-}$increases with a progression in skin irritation $[9$, $10]$.

An outlined square on the lateral lower leg $15 \mathrm{~cm}$ above the foot sole was taken as the measuring point. Therefore, the volunteer had to lay down comfortably on a patient bench to avoid perspiration and unwanted movements. After an acclimatization period of $20 \mathrm{~min}$, the measurement of skin hydration was repeated 4 times and the measurement of TEWL 2 times. All measurements were performed on the same anatomic area with constant room temperature $\left(23 \pm 0.5^{\circ} \mathrm{C}\right)$ and humidity in order to have reproducible data.

In order to define the leg volume as precisely as possible, measurements were performed by using Bodytronic ${ }^{\circledR} 600$ (Bauerfeind AG, Zeulenroda). The system is based on a digital video recording which is produced by projecting beams of light onto the participants' body. Therefore, the volunteer stands on a rotating platform. The acquired 3D image of the legs permits the exact calculation of the leg volume which is given in liters (L) [11] (Fig. 2).

Interface pressure measurements were performed by using $\mathrm{Pi}$ coPress $^{\circledR}$ (Microlab Elettronica, Italy). By means of 2 air-filled sensors which are placed between the lower leg (B1 level $-12 \mathrm{~cm}$ proximal to the inner ankle), compression stocking data were collected. Therefore, the volunteer had to be in a standing position with both

Influence of Compression Stockings on

Skin Hydration in Professionals
Table 5. Average and quartiles regarding skin hydration (in AU) within cohorts $\mathrm{AB}$ and $\mathrm{BA}$

\begin{tabular}{lccc}
\hline & Day 1 & Day 7 & Day 14 \\
\hline Mean value of AB & 32.28 & 24.36 & 25.81 \\
Median of AB & 34.14 & 25.74 & 25.34 \\
Lower quartile of AB & 28.18 & 21.08 & 22.41 \\
Upper quartile of AB & 36.38 & 27.64 & 29.21 \\
Mean value of BA & 28.81 & 28.06 & 22.89 \\
Median of BA & 28.80 & 27.70 & 24.88 \\
Lower quartile of BA & 25.36 & 24.38 & 19.74 \\
Upper quartile of BA & 32.27 & 31.74 & 26.04 \\
\hline
\end{tabular}

feet standing on the floor. The pressure was given in mmHg. Afterward, the collected data were filed and transferred to a computer via USB [12].

The assessment of stasis-induced complaints and wearing comfort was performed by means of questionnaires. The aim was to detect differences between the 2 types of compression hosiery. Therefore, different parameters as shown in Table 4 had to be evaluated. The participants were allowed to choose among the 4 qualities: none, mild, moderate, and severe. In the first wearing period as well as in the second wearing period, the volunteer received 7 questionnaires each. These had to be filled out every day. At the end of each week, the questionnaires were collected.

\section{Data Analysis and Statistics}

For an alpha value (significance level) of 5\% and a power of $80 \%$, a cohort size of $n=26$ was calculated. The mean value and the $95 \%$ confidence interval were calculated for all data depending on the different types of compression stockings which have been worn. To show absolute changes of the primary and secondary outcomes, sequence diagrams of the mean value have been applied. Furthermore, the measurements of the 2 wearing periods have been compared by match pair design with a paired $t$ test.

\section{Results}

\section{Terminology}

Because of the cross-over design, 2 cohorts named after the sequence of wearing periods 1 and 2 were built to represent the results. Cohort $\mathrm{AB}$ started with $\mathrm{MCS}$ and switched to MCS-SkC. Cohort BA started with MCS-SkC and switched to MCS.

\section{Participants}

The number needed to recruit was 26 . In total, 33 participants were enrolled in this cross-over trial. Because of noncompliance, 2 participants were excluded which means that 31 participants successfully finished the trial (Fig. 1). 
Fig. 3. Skin hydration - comparison of cohorts $\mathrm{AB}$ and $\mathrm{BA}$ (match pair design). Participants of cohort AB started to wear MCS for 7 days (days 1-7, highlighted in red) and switched to MCS-SkC (days 8-14, highlighted in green). Participants of cohort BA started with MCS-SkC (days 1-7, highlighted in green) and switched to MCS (days 8-14, highlighted in red).

Fig. 4. Skin hydration - delta graphics. Changes of skin hydration while wearing MCS and MCS-SkC. Respectively summarized for MCS and MCS-SkC using the results of wearing periods 1 and 2 in cohorts $\mathrm{AB}$ and $\mathrm{BA}$.
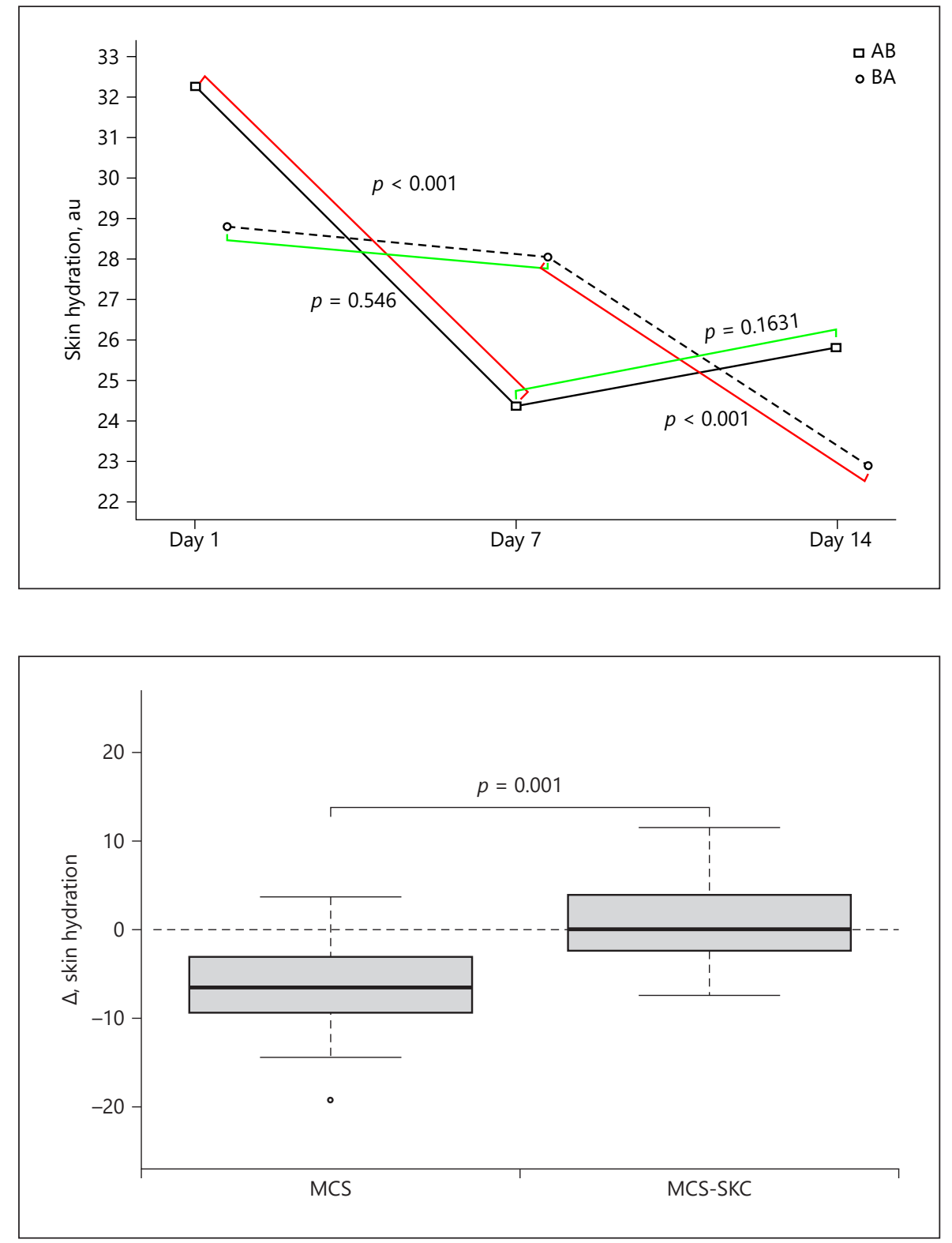

\section{Skin Hydration}

Table 5 shows the mean values, medians, and upper and lower quartiles regarding skin hydration before and after each wearing period with MCS and MCS-SkC in cohorts $\mathrm{AB}$ and $\mathrm{BA}$. The following graphics show the changes in skin hydration while wearing MCS and MCSSkC within wearing periods 1 and 2 in both cohorts $A B$ and $B A$. In cohort $A B$, there was a significant decrease regarding skin hydration after the wearing period with MCS $(p<0.001$; mean value before the wearing period $=$ 32.284 AU and mean value after the wearing period $=$ $24.363 \mathrm{AU})$. In contrast, there was a nonsignificant in- crease of skin hydration after the second wearing period with MCS-SkC ( $p=0.1631$; mean value before the wearing period $=24.363 \mathrm{AU}$ and mean value after the wearing period $=25.809 \mathrm{AU}$ ). In cohort $\mathrm{BA}$, there was a nonsignificant decrease regarding skin moisture after wearing period 1 with MCS-SkC ( $p=0.546$; mean value before the wearing period $=28.813 \mathrm{AU}$ and mean value after the wearing period $=28.063 \mathrm{AU}$ ). In contrast, there was a significant decrease regarding skin moisture after switching to wearing period 2 with MCS $(p<0.001$; mean value before the wearing period $=28.063 \mathrm{AU}$ and mean value after the wearing period $=22.892 \mathrm{AU}$ ) (Fig. 3). Figure 4
Mayer-Yousif/Konschake/Haase/Jünger/ Riebe 
Fig. 5. TEWL - comparison of cohorts $A B$ and BA (match pair design). Participants of cohort AB started to wear MCS for 7 days (days 1-7, highlighted in red) and switched to MCS-SkC (days 8-14, highlighted in green). Participants of cohort BA started with MCS-SkC (days 1-7, highlighted in green) and switched to MCS (days 8-14, highlighted in red). TEWL, transepidermal water loss.
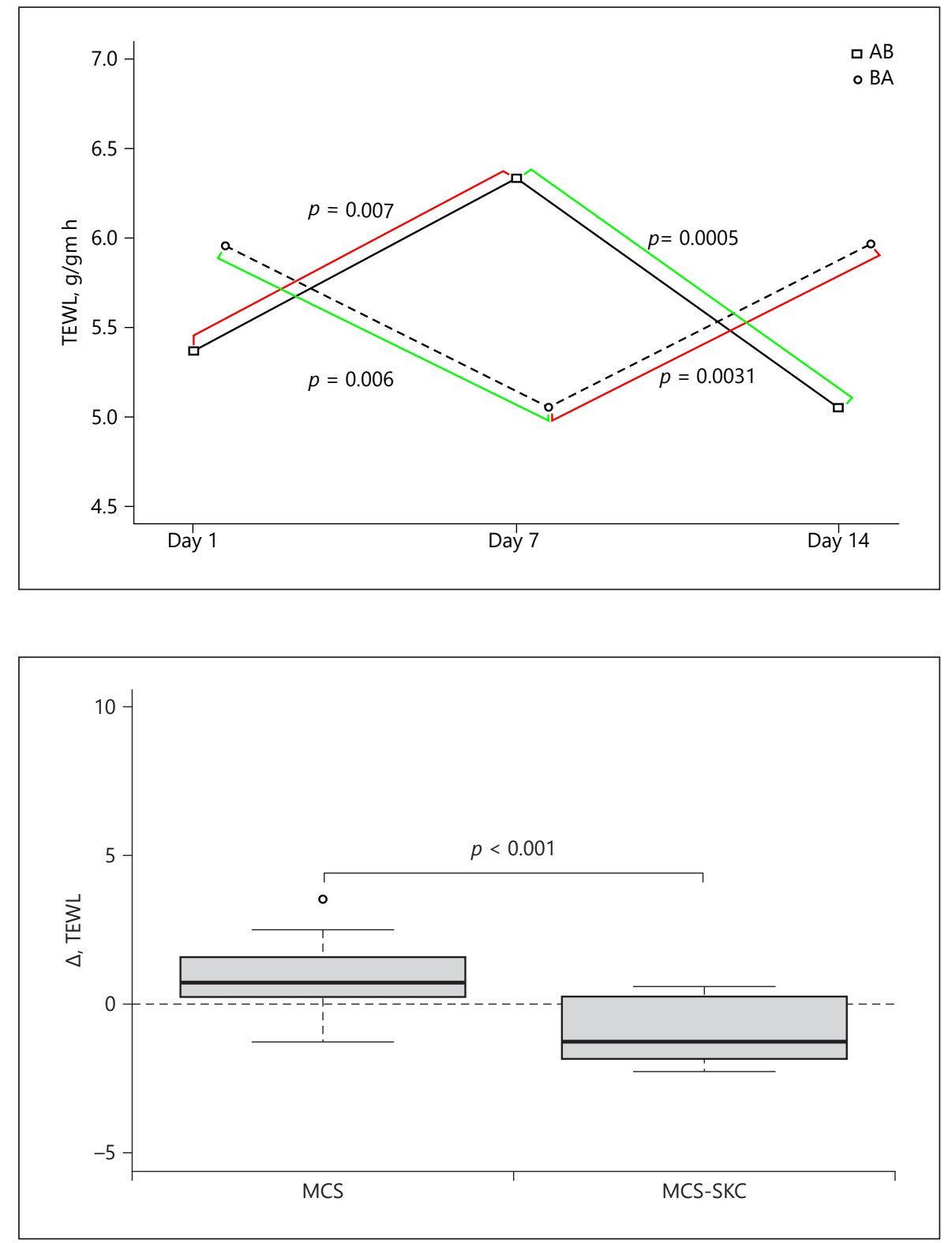

Fig. 6. TEWL - delta graphics. Changes of TEWL while wearing MCS and MCS-SkC. Respectively summarized for MCS and MCS-SkC using the results of wearing periods 1 and 2 in cohorts $A B$ and BA. TEWL, transepidermal water loss.
Table 6. Change of skin hydration while wearing MCS and MCSSkC, median and standard deviation

\begin{tabular}{lrl}
\hline & MCS & MCS-SkC \\
\hline Median & -8.3 & 0.3 \\
SD & 5.6 & 4.7 \\
\hline
\end{tabular}

Respectively summarized for both stocking types using the results of wearing periods 1 and 2 in cohorts $\mathrm{AB}$ and $\mathrm{BA}$. 
Table 7. Average and quartiles regarding TEWL (in $\mathrm{g} / \mathrm{m}^{2} \mathrm{~h}$ ) within cohorts $\mathrm{AB}$ and $\mathrm{BA}$

\begin{tabular}{llll}
\hline & Day 1 & Day 7 & Day 14 \\
\hline Mean value of AB & 5.37 & 6.33 & 5.05 \\
Median of AB & 5.09 & 5.76 & 5.14 \\
Lower quartile of AB & 4.42 & 5.43 & 4.45 \\
Upper quartile of AB & 6.32 & 7.23 & 5.65 \\
Mean value of BA & 5.95 & 5.05 & 5.96 \\
Median of BA & 5.56 & 4.85 & 5.56 \\
Lower quartile of BA & 5.25 & 4.36 & 5.06 \\
Upper quartile of BA & 6.66 & 5.75 & 6.86 \\
\hline
\end{tabular}

TEWL, transepidermal water loss.

Table 9. Average and quartiles regarding skin roughness (in $\mathrm{mm}^{3}$ ) within cohorts $\mathrm{AB}$ and $\mathrm{BA}$

\begin{tabular}{lccc}
\hline & Day 1 & Day 7 & Day 14 \\
\hline Mean value of AB & 15.42 & 18.80 & 18.00 \\
Median of AB & 15.78 & 18.92 & 17.56 \\
Lower quartile of AB & 14.18 & 16.90 & 15.44 \\
Upper quartile of AB & 16.67 & 20.71 & 20.56 \\
Mean value of BA & 16.52 & 16.43 & 19.12 \\
Median of BA & 16.27 & 16.91 & 19.04 \\
Lower quartile of BA & 14.12 & 15.00 & 16.76 \\
Upper quartile of BA & 18.92 & 17.86 & 21.48 \\
\hline
\end{tabular}

\section{Transepidermal Water Loss}

Table 7 shows the mean values, medians, and upper and lower quartiles regarding the TEWL before and after each wearing period with MCS and MCS-SkC in cohorts $\mathrm{AB}$ and $\mathrm{BA}$. TEWL is defined as $\mathrm{g} / \mathrm{m}^{2} \mathrm{~h}$. The following figures show the changes in TEWL while wearing MCS and MCS-SkC within wearing periods 1 and 2 in both cohorts $\mathrm{AB}$ and $\mathrm{BA}$. In cohort $\mathrm{AB}$, there was a significant increase regarding TEWL after the wearing period with $\operatorname{MCS}(p=0.007$; mean value before the wearing period $=$ $5.37 \mathrm{~g} / \mathrm{m}^{2} \mathrm{~h}$ and mean value after the wearing period $=$ $6.33 \mathrm{~g} / \mathrm{m}^{2} \mathrm{~h}$ ). In contrast, there was a significant decrease of TEWL after wearing period 2 with MCS-SkC $(p=$ 0.0005 ; mean value before the wearing period $=6.33 \mathrm{~g} / \mathrm{m}^{2}$ $\mathrm{h}$ and mean value after the wearing period $=5.05 \mathrm{~g} / \mathrm{m}^{2} \mathrm{~h}$ ). In cohort $\mathrm{BA}$, there was a significant decrease regarding TEWL after the wearing period with MCS-SkC $(p=0.006$; mean value before the wearing period $=5.95 \mathrm{~g} / \mathrm{m}^{2} \mathrm{~h}$ and mean value after the wearing period $=5.05 \mathrm{~g} / \mathrm{m}^{2} \mathrm{~h}$ ). In contrast, there was a significant increase of TEWL after the second wearing period with MCS ( $p=0.0031$; mean value before the wearing period $=5.05 \mathrm{~g} / \mathrm{m}^{2} \mathrm{~h}$ and mean
Table 8. Change of TEWL while wearing MCS and MCS-SkC, median and standard deviation

\begin{tabular}{llc}
\hline & MCS & MCS-SkC \\
\hline Median & 0.7 & -1.2 \\
SD & 1.2 & 1.1
\end{tabular}

Respectively summarized for both stocking types using the results of wearing periods 1 and 2 in cohorts $A B$ and BA. TEWL, transepidermal water loss.

value after the wearing period $=5.96 \mathrm{~g} / \mathrm{m}^{2} \mathrm{~h}$ ) (Fig. 5). To illustrate the effect of both compression stockings MCS and MCS-SkC on TEWL, delta graphics were used (Fig. 6). The graphic shows that wearing MCS leads to an increase of TEWL irrespective of its wearing period. Likewise, wearing MCS-SkC leads to a decrease of TEWL, again irrespective of its wearing period. Comparing both results of MCS and MCS-SkC regarding the effect on TEWL, there is a significant difference among the tested compression stockings with MCS-SkC maintaining a healthy skin barrier function. Furthermore, Table 8 shows the median and standard deviation, respectively, summarized for compression stockings MCS and MCS-SkC using the results of wearing periods 1 and 2 in cohorts $A B$ and BA.

\section{Skin Roughness}

Table 9 shows the mean values, medians, and upper and lower quartiles regarding skin roughness before and after each wearing period with MCS and MCS-SkC in cohorts $\mathrm{AB}$ and $\mathrm{BA}$. The following figures show the changes in skin roughness while wearing compression stockings $\mathrm{A}$ and $\mathrm{B}$ within wearing periods 1 and 2 in both cohorts $\mathrm{AB}$ and $\mathrm{BA}$. In cohort $\mathrm{AB}$, there was a significant increase of skin roughness after the wearing period with MCS ( $p=0.0015$; mean value before the wearing period $=15.42 \mathrm{~mm}^{3}$ and mean value after the wearing period $=18.8 \mathrm{~mm}^{3}$ ). In contrast, there was a nonsignificant decrease of skin roughness after the second wearing period with MCS-SkC $(p=0.4570$; mean value before the wearing period $=18.8 \mathrm{~mm}^{3}$ and mean value after the wearing period $=18.0 \mathrm{~mm}^{3}$ ). In cohort $\mathrm{BA}$, there was a nonsignificant decrease of skin roughness after wearing period 1 with MCS-SkC $(p=0.933$; mean value before the wearing period $=16.52 \mathrm{~mm}^{3}$ and mean value after the wearing period $=16.43 \mathrm{~mm}^{3}$ ). In contrast, there was a significant increase of skin roughness after the second wearing period with MCS ( $p=$ 
Fig. 7. Skin roughness - comparison of cohorts $\mathrm{AB}$ and $\mathrm{BA}$ (match pair design). Participants of cohort AB started to wear MCS for 7 days (days 1-7, highlighted in red) and switched to MCS-SkC (days 8-14, highlighted in green). Participants of cohort BA started with MCS-SkC (days 1-7, highlighted in green) and switched to MCS (days 8-14, highlighted in red).
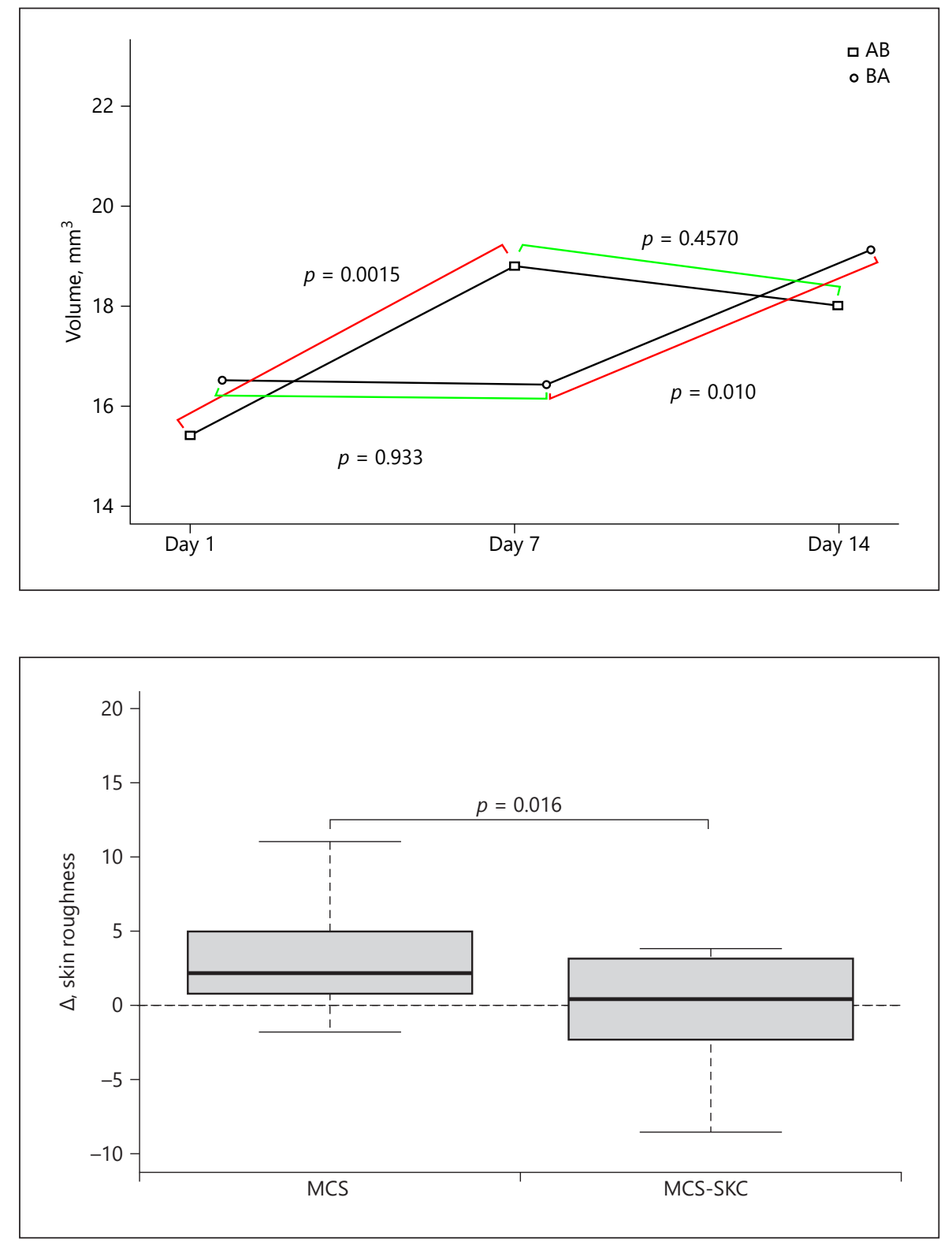

Fig. 8. Skin roughness - delta graphics. Changes of skin roughness while wearing MCS and MCS-SkC. Respectively summarized for MCS and MCS-SkC using the results of wearing periods 1 and 2 in cohorts $\mathrm{AB}$ and $\mathrm{BA}$.
0.010 ; mean value before the wearing period $=16.43$ $\mathrm{mm}^{3}$ and mean value after the wearing period $=19.12$ $\mathrm{mm}^{3}$ ) (Fig. 7). Figure 8 shows the influence of compression stockings MCS and MCS-SkC on skin roughness using delta graphics. The results of delta graphics show that there is a significant difference between compression stockings MCS and MCS-SkC $(p=0.016)$. The figures illustrate that wearing MCS causes an increase of skin roughness irrespective of its wearing period while wearing MCS-SkC keeps the normal skin barrier function stable. These results are also illustrated by Table 10 which shows the median and standard deviation, re-
Table 10. Change of skin roughness while wearing MCS and MCSSkC, median and standard deviation

\begin{tabular}{lll}
\hline & MCS & MCS-SkC \\
\hline Median & 2.2 & 0.4 \\
SD & 3.5 & 4.0 \\
\hline
\end{tabular}

Respectively summarized for both stocking types using the results of wearing periods 1 and 2 in cohorts $\mathrm{AB}$ and $\mathrm{BA}$. 
Fig. 9. Leg volume - comparison of cohorts $\mathrm{AB}$ and $\mathrm{BA}$ (match pair design). Participants of cohort AB started to wear MCS for 7 days (days 1-7, highlighted in red) and switched to MCS-SkC (days 8-14, highlighted in green). Participants of cohort BA started with MCS-SkC (days 1-7, highlighted in green) and switched to MCS (days 8-14, highlighted in red).

Fig. 10. Leg volume - delta graphics. Changes of skin roughness while wearing MCS and MCS-SkC. Respectively summarized for MCS and MCS-SkC using the results of wearing periods 1 and 2 in cohorts $\mathrm{AB}$ and $\mathrm{BA}$.
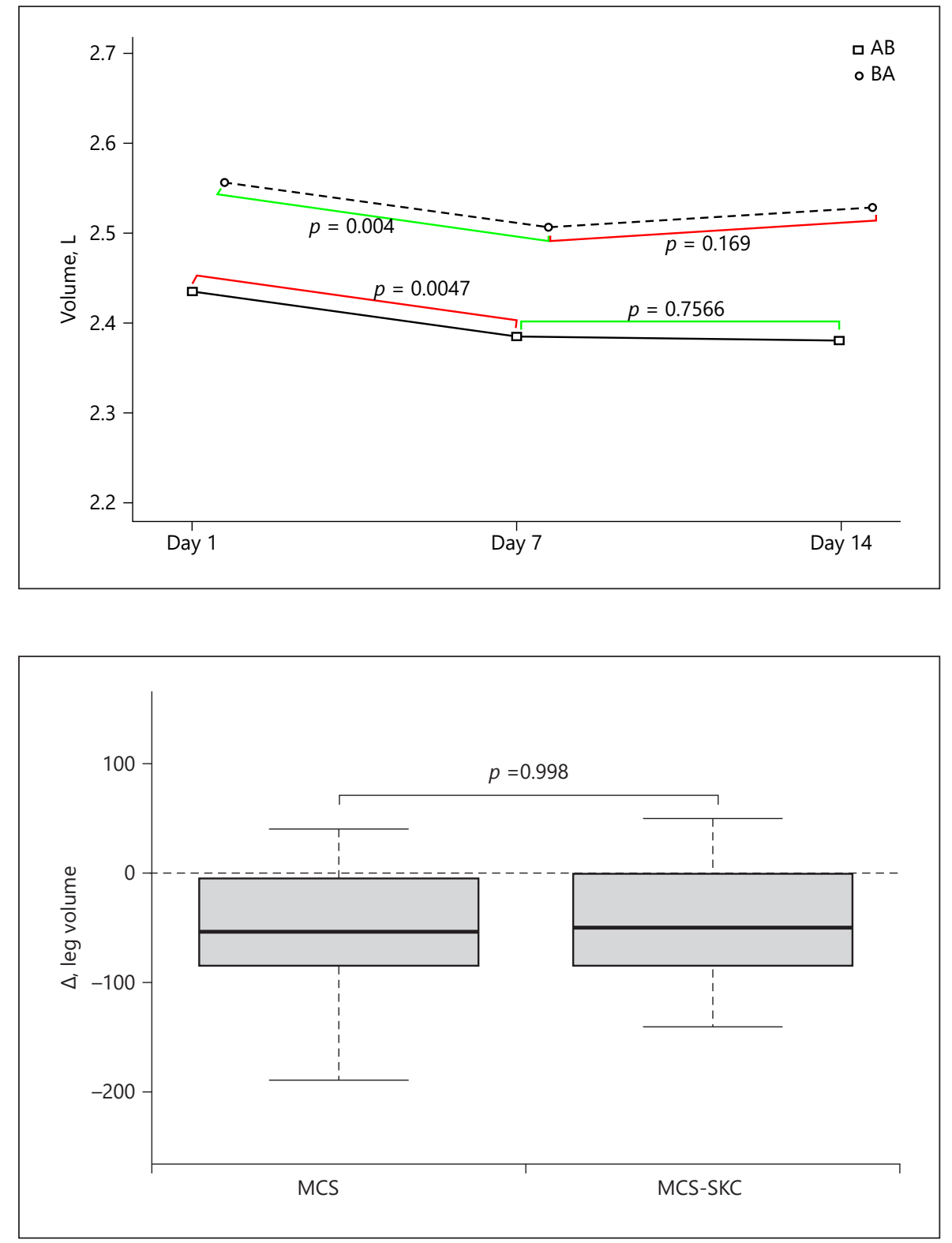

spectively, summarized for compression stockings MCS and MCS-SkC using the results of wearing periods 1 and 2 in cohorts $\mathrm{AB}$ and $\mathrm{BA}$.

\section{Leg Volume}

Table 11 shows the descriptive statistics as to leg volume of cohorts $\mathrm{AB}$ and $\mathrm{BA}$ at days 1, 7, and 14. The following figures show the changes in leg volume while wearing compression stockings $\mathrm{A}$ and $\mathrm{B}$ within wearing periods 1 and 2 in both cohorts $\mathrm{AB}$ and $\mathrm{BA}$. Both MCS and MCS-SkC resulted in a significant reduction of leg volume after the first wearing period in cohorts $\mathrm{AB}(p=$
$0.004)$ and BA $(p=0.0047)$. In contrast, there were no significant changes regarding leg volume in the second wearing period, neither in cohort $\mathrm{AB}$ nor $\mathrm{BA}$. In cohort $A B$, there was a nonsignificant increase of leg volume after switching to compression stocking $B(p=0.169)$. In cohort BA, there was a nonsignificant decrease of leg volume after switching to compression stocking A ( $p=$ 0.7566) (Fig. 9). Delta graphics show that there was a clear reduction in leg volume irrespective of the wearing period while wearing MCS as well as MCS-SkC (Fig. 10). Thus, a significant difference could not be shown between both compression stockings. This is also illustrated by Table 12 
Fig. 11. Interface pressure - comparison of cohorts $\mathrm{AB}$ and $\mathrm{BA}$ (match pair design). Participants of cohort $A B$ started to wear MCS for 7 days (days 1-7, highlighted in red) and switched to MCS-SkC (days 8-14, highlighted in green). Participants of cohort BA started with MCS-SkC (days 1-7, highlighted in green) and switched to MCS (days 8-14, highlighted in red).

Fig. 12. Interface pressure - delta graphics. Changes of skin roughness while wearing MCS and MCS-SkC. Respectively summarized for MCS and MCS-SkC using the results of wearing periods 1 and 2 in cohorts $\mathrm{AB}$ and $\mathrm{BA}$.
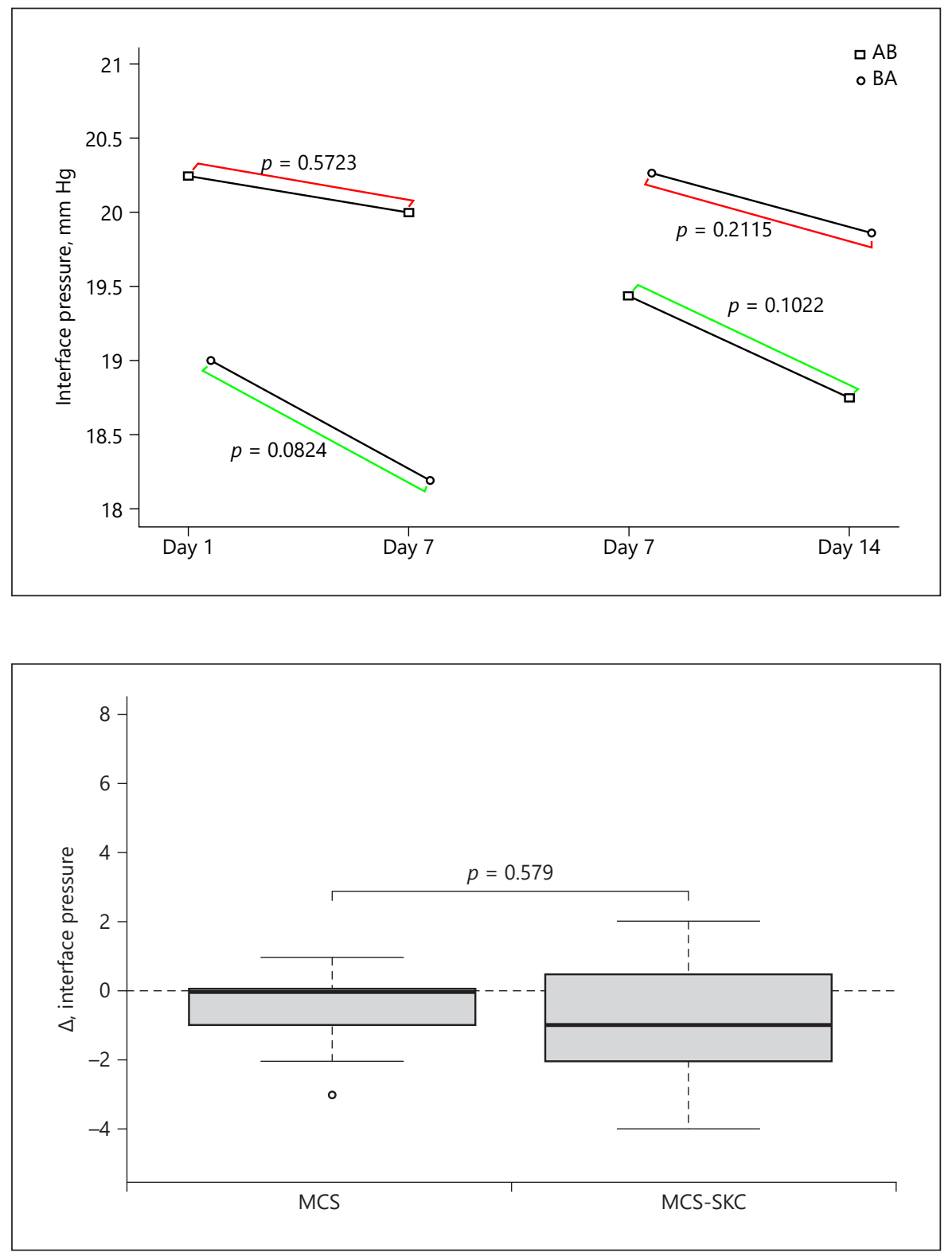

Table 11. Average and quartiles regarding leg volume (in L) within cohorts $\mathrm{AB}$ and $\mathrm{BA}$

\begin{tabular}{llll}
\hline & Day 1 & Day 7 & Day 14 \\
\hline Mean value of AB & 2.44 & 2.39 & 2.38 \\
Median of AB & 2.42 & 2.35 & 2.38 \\
Lower quartile of AB & 2.21 & 2.17 & 2.17 \\
Upper quartile of AB & 2.66 & 2.60 & 2.60 \\
Mean value of BA & 2.56 & 2.51 & 2.53 \\
Median of BA & 2.42 & 2.36 & 2.36 \\
Lower quartile of BA & 2.18 & 2.13 & 2.14 \\
Upper quartile of BA & 2.94 & 2.89 & 2.92 \\
\hline
\end{tabular}

Table 12. Change of leg volume while wearing MCS and MCSSkC, median and standard deviation

\begin{tabular}{lrc}
\hline & MCS & MCS-SkC \\
\hline Median & -55.0 & -50.0 \\
SD & 59.6 & 56.5 \\
\hline
\end{tabular}

Respectively summarized for both stocking types using the results of wearing periods 1 and 2 in cohorts $\mathrm{AB}$ and $\mathrm{BA}$. 
Table 13. Average and quartiles regarding interface pressure (in $\mathrm{mm} \mathrm{Hg}$ ) within cohorts $\mathrm{AB}$ and $\mathrm{BA}$

\begin{tabular}{lllll}
\hline & $\begin{array}{l}\text { Start first } \\
\text { period }\end{array}$ & $\begin{array}{l}\text { End first } \\
\text { period }\end{array}$ & $\begin{array}{l}\text { Start second } \\
\text { period }\end{array}$ & $\begin{array}{l}\text { End second } \\
\text { period }\end{array}$ \\
\hline Mean value of AB & 20.2 & 20.0 & 19.4 & 18.8 \\
Lower quartile of AB & 19.1 & 18.8 & 18.1 & 17.5 \\
Upper quartile of AB & 21.4 & 21.2 & 20.7 & 20.0 \\
Mean value of BA & 19.0 & 18.2 & 20.3 & 19.9 \\
Lower quartile of BA & 17.8 & 17.0 & 19.3 & 19.0 \\
Upper quartile of BA & 20.2 & 19.4 & 21.2 & 20.8 \\
\hline
\end{tabular}

Table 14. Change of interface pressure while wearing MCS and MCS-SkC, median and standard deviation

\begin{tabular}{lrc}
\hline & MCS & MCS-SkC \\
\hline Median & -0.5 & -1.0 \\
SD & 1.7 & 1.7 \\
\hline
\end{tabular}

Respectively summarized for both stocking types using the results of wearing periods 1 and 2 in cohorts $\mathrm{AB}$ and $\mathrm{BA}$.

which shows the median and standard deviation, respectively, summarized for compression stockings MCS and MCS-SkC using the results of wearing periods 1 and 2 in cohorts $\mathrm{AB}$ and $\mathrm{BA}$.

\section{Interface Pressure}

Table 13 shows the mean values and upper and lower quartiles regarding interface pressure before and after each wearing period with MCS and MCS-SkC in cohorts $\mathrm{AB}$ and $\mathrm{BA}$. The following figures show the changes in interface pressure while wearing compression stockings $A$ and $B$ within wearing periods 1 and 2 in both cohorts $\mathrm{AB}$ and $\mathrm{BA}$. All values before starting and after ending each wearing period are listed. MCS as well as MCS-SkC showed a nonsignificant reduction of interface pressure in both wearing periods 1 and 2 regardlessly belonging to cohort $\mathrm{AB}$ or $\mathrm{BA}$. In cohort $\mathrm{AB}$, wearing MCS resulted in a decrease of interface pressure of $p=0.5723$, whereas wearing MCS-SkC resulted in a decrease of $p=$ 0.1022 . Likewise, the decrease of interface pressure in cohort BA was defined with $p=0.0824$ with reference to MCS-SkCand $p=0.2115$ with reference to MCS (Fig. 11). Also, delta graphics show that there was no significant difference in the change of interface pressure regardlessly of wearing MCS or MCS-SkC ( $p=0.579)$ (Fig. 12). Both compression stockings had a mild loss of interface pressure at the end of each wearing period. This shows that integrating a care emulsion within MCS-SkC does not have any impact on its efficacy as compression stocking. Again, Table 14 illustrates this result in showing the median and standard deviation, respectively, summarized for compression stockings MCS and MCS$\mathrm{SkC}$ using the results of wearing periods 1 and 2 in cohorts $\mathrm{AB}$ and $\mathrm{BA}$.

\section{Complaints}

In order to identify the most common complaints during compression therapy, the patients were asked to evaluate various parameters (Table 4 ) which were analyzed as shown in Figure 13. The graphic illustrates the 6 daily differences (delta) for the summed and averaged results of all patients for each compression stocking MCS and MCS-SkC irrespective of their wearing time in relation to the initial day. The low severity of the complaints demonstrates the high quality level of both compression stockings; nevertheless, there are significant differences between the averaged and summarized complaints. Overall, the development of complaints by wearing MCS-SkC (dashed line) is significantly different from the rating of the complaints by wearing conventional MCS (solid line), and the wearing comfort of MCS-SkC was significantly rated better than MCS $(p=0.017)$. One of the complaints asked was the assessment of the roughness of the skin while wearing the respective compression stocking.

In another graph (Fig. 14), the daily severity of the skin roughness is plotted for both patient groups depending on the stocking worn. For cohort AB, in which MCS was worn first, there is a daily increasing subjective feeling of roughness. This increase extends into the second wearing phase (wearing phase of MCS-SkC) and has its peak on day 8 (first day of wearing MCS-SkC). This increase up to the second wearing phase can be explained by the disturbed skin barrier while wearing the first stocking and corresponds to an overhang from this first wearing phase. MCS-SkC can compensate this skin barrier disorder
Mayer-Yousif/Konschake/Haase/Jünger/ Riebe 
Fig. 13. The development of complaints regarding the wearing comfort of 2 different types of socks. For this purpose, the evaluation of the first day for each subject is subtracted from the evaluation of the 6 consecutive days for each individual item. It should be noted that the data of the stocking change on day 8 (wear to day 14) were integrated into the presentation, so day 8 then corresponds to day 1 . The differences are summed up for each day divided by the number of participants of the respective stocking type group. This results in 6 average values for each stocking type are displayed with a line chart.

Fig. 14. Detailed presentation of the 4-level daily subjective assessment of skin roughness over the entire period of both wearing phases. The inputs of all patients were summed up and averaged. Cohort AB: MCS was worn first, and there is a daily increasing subjective feeling of roughness. The increase up to the second wearing phase (day $8=$ first day of wearing phase MCS-SkC) can be explained by the disturbed skin barrier while wearing the first stocking and corresponds to an overhang from this first wearing phase. MCS-SkC can compensate this skin barrier disorder which is shown by the stable and not increasing values from day 9. Cohort BA: MCS-SkC was worn first, and feeling of skin roughness remains stable and increases only in the second wearing phase (wearing phase of MCS). The differences in both groups are not significant on most days, and day 9 shows a significant difference in both groups and represents in each cohort the overhang from the previous wearing phase.

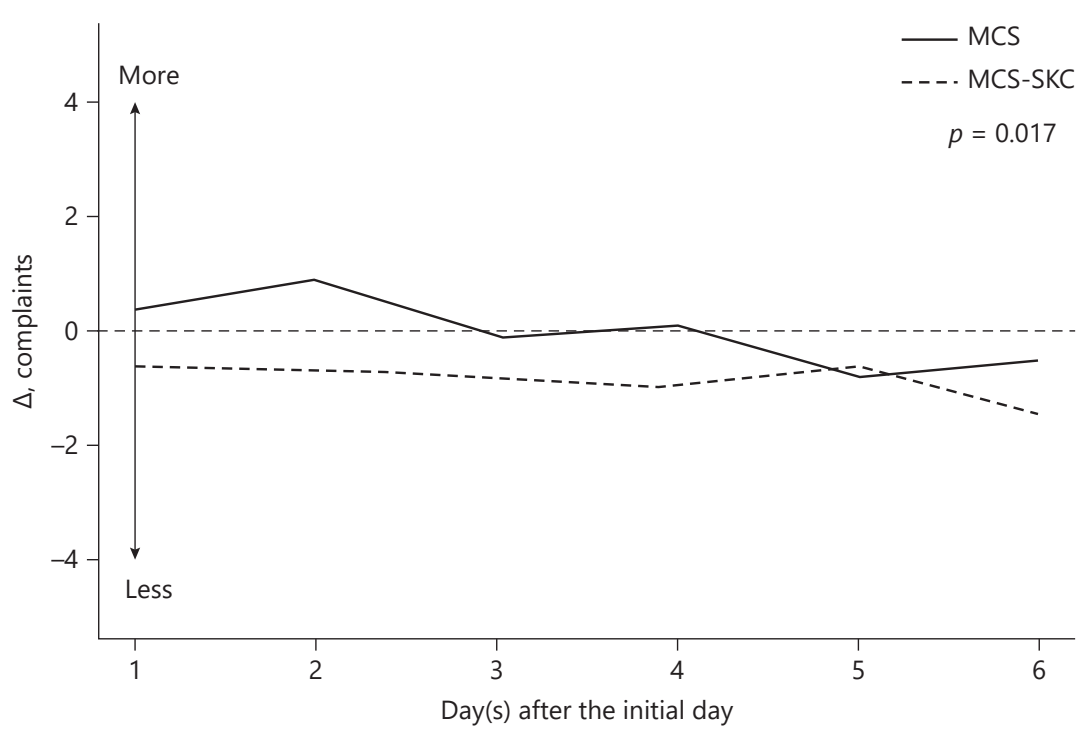

Day(s) after the initial day

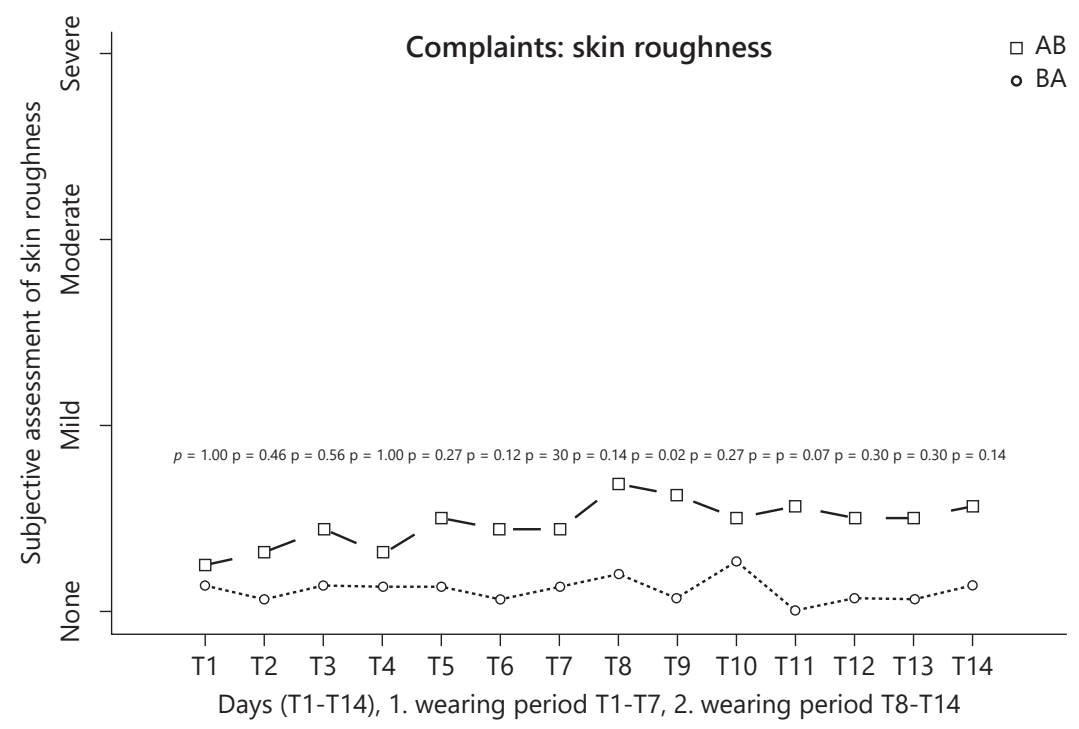

which is shown by the stable and not increasing values from day 9.

In the BA cohort, in which MCS-SkC was worn first, the feeling of skin roughness remains stable and increases only in the second wearing phase (wearing phase of MCS), once on day 10 and continuously from day 11 . The differences in both groups are not significant on most days due

to the high quality level of both stockings. But, day 9 shows a significant difference in both groups and represents in each cohort the overhang from the previous wearing phase and the associated barrier condition of the skin. This detailed representation of a very meaningful complaint can be supplemented and supported by clinical photos of the participants (Fig. 15, 16). 
Fig. 15. Comparison pictures of a participant before (a) and after (b) 7 days of wearing medical compression stocking without integrated care (MCS, stocking type A). Clinically there is a pronounced fine lamellar scaling after 7 days of wear (b), and this is a sign of a disturbed skin barrier and corresponds to an increasing drying out of the skin using MCS.

Fig. 16. Comparison pictures of a participant before (a) and after (b) 7 days of wearing medical compression stocking with integrated care (MCS-SkC, stocking type B). Clinically, the skin surface is stable without any signs of drying out of the skin, and this corresponds to a stable skin barrier function using MCS-SkC.
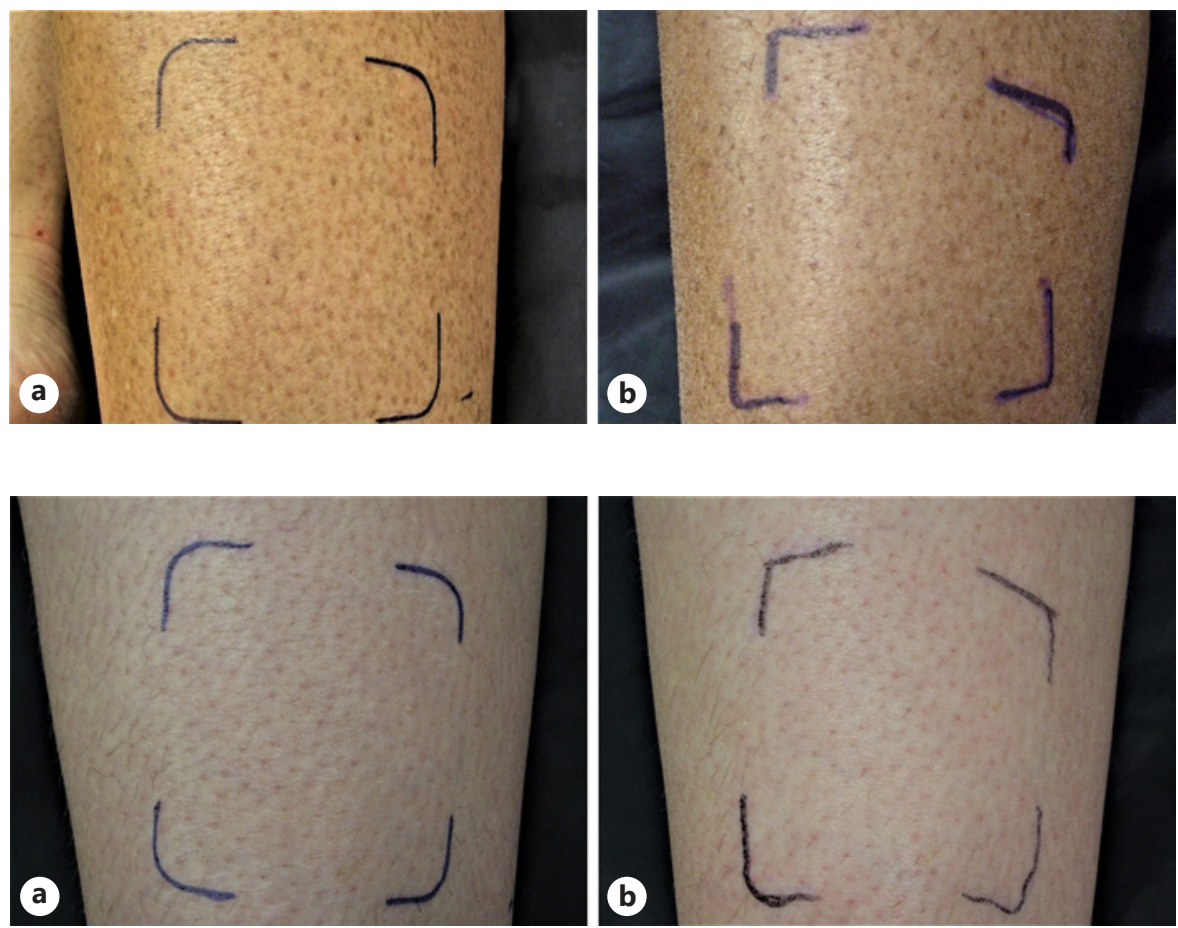

\section{Discussion}

The aim of this trial was to show the effect of different compression materials upon human skin barrier function comprising skin moisture as primary as well as TEWL, skin roughness, leg volume, and interface pressure as secondary outcomes. Therefore, only healthy people with occupational leg edema were included. In order to minimize confounding variables, a randomized cross-over study design was chosen in which every participant acted as their own control. As it is already shown in former studies, the usage of compression hosiery not only has an essential influence on human skin but also interferes with the compliance of affected persons $[3,13]$. Thus, it is necessary to develop adequate compression materials which cause less adverse events but obtain the natural skin barrier function in order to improve compression therapy. Within this randomized cross-over trial, 2 different stockings, MCS and care-emulsionequipped MCS-SkC, have been tested and directly compared by means of different measurement methods. The results of this cross-over trial showed that general compression stockings like MCS not only have a negative effect on skin hydration but also on TEWL and skin roughness. Skin moisture was significantly reduced in both cohorts $\mathrm{AB}$ and $\mathrm{BA}$ after wearing MCS, regardless of whether starting with MCS first or switching to it after wearing MCS-SkC. Furthermore, TEWL and skin roughness were increased after wearing MCS. Regarding TEWL, this effect was significant in cohorts $A B$ as well as BA, no matter wearing MCS first or after MCS-SkC. Likewise, there was a significant effect after wearing MCS in both cohorts regarding skin roughness. All 3 parameters indicate a temporary malfunction due to skin defects created by the usage of MCS. Likewise, the study showed that MCS-SkC had a positive effect on skin hydration which could be obtained. Regarding TEWL, there was a significant decrease in cohort $\mathrm{AB}$ as well as cohort BA after wearing MCS-SkC. Furthermore, skin roughness was nonsignificantly reduced wearing MCS$\mathrm{SkC}$ in both cohorts $\mathrm{AB}$ and $\mathrm{BA}$. These parameters show that the integrated care emulsion preserves the integrity of human skin. Literature research shows that there are yet less comparative studies. Those few studies available investigate the change of skin barrier function in CVI patients as, for example, the study conducted in 2011 by Riebe [13]. In a parallel group design, it could be shown that the parameters skin moisture, TEWL, and skin roughness were positively influenced by wearing a compression stocking equipped with care emulsion which is comparable to our findings [13]. Another randomized controlled study from 2015 by Thormählen et al. [14] as- 
sessed the effect of skin care during compression therapy of persons with and without chronic venous diseases. The trial showed that here also the application of care emulsion could contribute to the maintenance of a normal skin barrier function while excluding it led to a significant reduction of skin moisture as well as a significant increase of TEWL and skin roughness in both groups [14]. These results indicate that skin health basically depends on skin care no matter integrating it into a compression stocking or applying it self-contained. The care emulsion used in this study is composed of the main ingredients urea, vitamin E, and natural oils. As a component of the NMF, urea is responsible to store water within the stratum corneum and thus keeps its integrity and elasticity [15]. In contrast, a lack of NMF is linked to xerosis as well as different skin diseases as could be shown in many studies [16-18]. In addition, an unimpaired skin barrier is necessary in order to avoid excessive evaporation of water. For this, the intercellular spaces of human skin are filled with lipids which is why natural oils are another ingredient of the integrated care emulsion used in MCS-SkC [19]. Vitamin E (tocopherol) is a natural antioxidant which keeps the lipids within the stratum corneum stable and prevents them from oxidation. This, too, ensures a healthy skin barrier function and maintains the skin moisturized [20].

As secondary outcomes, leg volume and interface pressure were determined. Using a compression therapy belonging to Ccl. 1, this randomized cross-over trial exclusively examined healthy persons with occupational (= orthostatic) leg edema. It could be shown that leg edema and life quality are directly linked to each other and thus justifies compression therapy in healthy individuals. By reducing leg edema and with it somatic as well as psychic complaints, the application of Ccl. 1 stockings is highly recommended within this group. A study performed in 2008 by Blättler et al. [21] shows that an average pressure of $15-20 \mathrm{~mm} \mathrm{Hg}$ was able to lead to a significant reduction of leg edema $(54.9 \mathrm{~mL})$ which is comparative to our findings. According to Blättler et al. [21], a higher pressure is not of any additional benefit and increases discomfort. With an average pressure of 19-20 $\mathrm{mm} \mathrm{Hg}$, this cross-over trial showed an average volume reduction of 49-50 mL in the first period of both cohorts $A B$ and BA. Similar results can also be seen in further studies [22]. Nevertheless, the quoted studies did not investigate different types of compression stockings as it was performed in this cross-over trial. Comparing MCS and MCS-SkC, there were no significant differences between both types of compression stockings. Both wearing periods resulted

Influence of Compression Stockings on

Skin Hydration in Professionals in a significant reduction of leg volume, regardless of wearing compression stocking A or B. Likewise, there was a nonsignificant reduction of interface pressure after wearing periods 1 and 2, regardless of wearing compression stocking A or B. This means that the integrated care emulsion of MCS-SkC does not have any disadvantageous effects concerning its effectiveness as compression material. Regarding stasis-induced complaints and the influence of compression therapy on daily life, literature research reveals several studies. Many of them deal with complaints occurring in patients with chronic venous diseases but also those in objectively healthy people. The main problems despite leg swelling as to the quoted studies are feeling of heaviness and tension. Although those stasis-induced complaints can be significantly reduced with medical stockings, compression therapy is often rejected by individuals due to impaired skin conditions like dryness and itchiness $[1,2,13,21-30]$. In this trial, the participants were asked about their complaints every day while wearing compression stockings A and B. Therefore, questionnaires were handed out and analyzed in the end. It could be shown that both types, MCS and MCS-SkC, had a high wearing comfort. All parameters which had to be evaluated were noted as "nonexistent" or "mild" complaints. But, despite these low severity of complaints by wearing both types of compression stockings, there is a significant difference in the averaged complaints in favor of MCS-SkC. Thus, the experimentally verified benefit of the integrated care emulsion of MCS-SkC could also be attested subjectively, and there were no adverse reactions either.

\section{Limitations and Conclusion}

The results of this study refer to a compression therapy with MCS and MCS-SkC, manufactured by Bauerfeind $\mathrm{GmbH}$, and cannot be transferred to the usage of other compression material. During the trial, participants were not allowed to use regular skin care emulsions at all. The dehydration effect seen during compression therapy may be minimized by the regular application of skin care as seen in the study of Thormählen et al. [14]. The participants of this trial were included in a 14-day test period which means an ongoing alteration in temperature and humidity and thus changes regarding the individual skin condition even if the investigations were performed at standardized conditions. Furthermore, we urged the participants to maintain daily activity at examination days as similar as possible. Anyways bias concerning those fac- 
tors cannot be completely excluded. As the 2 test periods were performed consecutively, it could be possible that the effect of the first test period had an impact onto the second test period. This means that by wearing MCS first, the nurturing effect of MCS-SkC could be minimized. In contrast, by wearing MCS-SkC first, the negative effect of MCS could be attenuated.

It could be shown that both compression stockings, MCS and MCS-SkC, had good results concerning its effectiveness as compression hosiery by receiving a decrease of leg volume and retaining its interface pressure. This study showed that MCS had a negative effect on skin moisture, TEWL, and skin roughness. This effect might have been aggravated due to the fact that participants were not allowed to use any kind of additional care emulsion during the wearing period. However, the care-emulsion-equipped MCS-SkC obtained skin moisture and had a positive effect on TEWL as well as skin roughness. This leads to the conclusion that both compression stockings are appropriate devices in compression therapy with MCS-SkC being eligible to maintain the human skin barrier function during its application and thus to improve the wear comfort.

\section{Statement of Ethics}

The study was conducted at the Department of Dermatology at the University of Greifswald, and an approval of the local ethics committee was given (internal registration number: BB 082/16) according to the principles of the Declaration of Helsinki.

\section{Conflict of Interest Statement}

The authors declare no potential conflicts of interest with respect to the research, authorship, and/or publication of this article.

\section{Funding Sources}

The authors received no financial support for the research, authorship, and/or publication of this article.

\section{Author Contributions}

M.J. and H.R. conceptualized the study. M.M.-Y. and H.R. were responsible for the performance of the measurements and data collection. H.H. conducted the statistical analyses. A critical revision of the manuscript was performed by M.J., H.R. and W.K. All authors contributed to the accomplishment of the manuscript by reading and continually improving it.

\section{References}

1 Rabe E, Pannier-Fischer F, Bromen K, Schuldt K, Stang A, Poncar Ch, et al. Bonner Venenstudie der Deutschen Gesellschaft für Phlebologie. Epidemiologische Untersuchung zur Frage der Häufigkeit und Ausprägung von chronischen Venenkrankheiten in der städtischen und ländlichen Wohnbevölkerung. Phlebology. 2003;32:1-14.

2 Blazek C, Amsler F, Blaettler W, Keo HH, Baumgartner I, Willenberg T. Compression hosiery for occupational leg symptoms and leg volume: a randomized crossover trial in a cohort of hairdressers. Phlebology. 2013; 28(5):239-47.

3 Reich-Schupke S, Murmann F, Altmeyer P, Stücker M. Quality of life and patients' view of compression therapy. Int Angiol. 2009; 28(5):385-93.

4 Verdier-Sévrain S, Bonté F. Skin hydration: a review on its molecular mechanisms. J Cosmet Dermatol. 2007;6(2):75-82.

5 Rawlings AV, Harding CR. Moisturization and skin barrier function. Dermatol Ther. 2004;17(Suppl 1):43-8.

6 Lüllmann-Rauch R. Taschenbuch histologie, 5. Auflage. Stuttgart: Georg Thieme Verlag; 2015.

7 Courage \& Khazaka GmbH. Informationen und Gebrauchsanweisung zum Corneometer ${ }^{\circledR}$ CM 825. Köln: Courage \& Khazaka $\mathrm{GmbH} ; 2013$
8 Courage \& Khazaka GmbH. Informationen und Gebrauchsanweisung zum Tewameter TM 300. Köln: Courage \& Khazaka GmbH; 2014.

9 Piche E, Häfner HM, Hoffmann J, Jünger M. FOITS (fast optical in vivo topometry of human skin): new approaches to 3 -D surface structures of human skin. Biomed Tech. 2000; 45(11):317-22.

10 Breuckmann B. Grundlagen und Anwendungen der 3D-Bildverarbeitung. Meersburg: Breuckmann GmbH; 2003.

11 Bauerfeind $^{\otimes} \mathrm{GmbH}$. Bodytronic ${ }^{\odot}$ 600: Messtechnik der nächsten Generation (2015). Available from: https://www.bauerfeind.de/ $\mathrm{de} /$ themen/handel-technik/digitalemesstechnik/bodytronic600.html (Stand: 2017 Sep 3).

12 Microlab Elettronica S.a.s. di Bergamo Giorgio \& C. Technical manual for users M-688 Rev. 5. Ponte S. Nicolo' (PD) Italy: Microlab Elettronica S.a.s, 2012.

13 Riebe H. Einfluss medizinischer Kompessionsstrümpfe auf die Barrierefunktion der Haut bei Patienten mit Chronischer Venöser Insuffizienz (CVI). Dissertation zur Erlangung des akademischen Grades Doktor der Medizin; 2011.
14 Thormählen H, Riebe H, Jünger M. Wirksamkeit von Hautpflege bei der Kompressionstherapie. Bauerfeind Doktorandenpreis, DGP-Tagung 2015 in Bamberg. Available from: https://www.bauerfeind.de/de/themen/medizin-wissenschaft/doktorandenprogramm.html?utm_source $=$ shortlink \& utm_medium $=$ redirect $\& \quad$ utm campaign=shortlink_doktorandenprogramm (Stand: 2017 Dec 1).

15 Fowler J. Understanding the natural moisturizing factor in skin hydration. Practical Dermatol. 2012;36-40.

16 Horii I, Nakayama Y, Obata M, Tagami H. Stratum corneum hydration and amino acid content in xerotic skin. Br J Dermatol. 1989; 121(5):587-92.

17 Denda M, Hori J, Koyama J, Yoshida S, Nanba R, Takahashi $M$, et al. Stratum corneum sphingolipids and free amino acids in experimentally-induced scaly skin. Arch Dermatol Res. 1992;284(6):363-7.

18 Harding CR, Watkinson A, Rawlings AV, Scott IR. Dry skin, moisturization and corneodesmolysis. Int J Cosmet Sci. 2000;22(1): 21-52.

19 Rawlings AV, Scott IR, Harding CR, Bowser PA. Stratum corneum moisturization at the molecular level. J Invest Dermatol. 1994; 103(5):731. 
20 Traber MG, Atkinson J. Vitamin E, antioxidant and nothing more. Free Radic Biol Med. 2007;43(1):4-15.

21 Blättler W, Kreis N, Lun B, Winiger J, Amsler F, et al. Leg symptoms of healthy people and their treatment with compression hosiery. Phlebology. 2008;23(5):214-21.

22 Partsch H, Winiger J, Lun B. Compression stockings reduce occupational leg swelling. Dermatol Surg. 2004;30(5):737-43.

23 Tschopp SC. [Beinbeschwerden. Subjektive Beschwerden und objektive Befunde. Ergebnisse des Basler Venenprojektes, Follow-up III]. Dissertation Universitaet Basel; 1990.
24 Amsler F, Blättler W. Compression therapy for occupational leg symptoms and chronic venous disorders: a meta-analysis of randomised controlled trials. Eur J Vasc Endovasc Surg. 2008;35(3):366-72.

25 Streeten DH. Idiopathic edema. Pathogenesis, clinical features, and treatment. Endocrinol Metab Clin North Am. 1995 Sep;24(3): 531-47.

26 Cavanaugh RMJr. Orthostatic edema in adolescents: more than walking on water. Pediatr Rev. 2005 Apr;26(4):115-24.

27 Carpentier PH, Poulain C, Fabry R, Chleir F, Guias B, Bettarel-Binon C. Ascribing leg symptoms to chronic venous disorders: the construction of a diagnostic score. J Vasc Surg. 2007;46(5):991-6.
28 Kendler M, Haas E. [Subjective venous symptoms: review and presentation of a pilot study]. Phlebologie. 2006;35:19-23.

29 Ruckley CV, Evans CJ, Allan PL, Lee AJ, Fowkes FG. Chronic venous insufficiency: clinical and duplex correlations. The Edinburgh Vein Study of venous disorders in the general population. J Vasc Surg. 2002;36:520 5 .

30 Bradbury A, Evans C, Allan P, Lee A, Ruckley $\mathrm{CV}$, Fowkes FG. What are the symptoms of varicose veins? Edinburgh Vein Study crosssectional population survey. BMJ. 1999;318: 353-6. 